\title{
Prevalence and Correlates of Electronic Cigarette Use Among U.S. Adult Survivors of Adolescent and Young Adult Cancer
}

\author{
Godfred O Antwi ${ }^{*}$; David K. Lohrmann ${ }^{2}$; Wasantha Jayawardene ${ }^{2,3}$; Cecilia S Obeng ${ }^{2}$ \\ ${ }^{1}$ Department of Public Health and Health Education, The College at Brockport, State University of New York, Brockport, NY 14420, \\ USA
}

${ }^{2}$ Department of Applied Heath Science, Indiana University School of Public Health Bloomington, IN 47405, USA

${ }^{3}$ Institute for Research on Addictive Behavior, Indiana University School of Public Health Bloomington, IN, USA

\author{
*Corresponding Author(s): Godfred O Antwi \\ Department of Public Health and Health Educa- \\ tion, The College at Brockport, State University \\ of New York, Brockport, NY 14420, USA \\ Tel: 585-395-5145, Fax: 585-3955-246; \\ Email: gantwi@brockport.edu
}

Received: Mar 03, 2020

Accepted: Apr 17, 2020

Published Online: Apr 23, 2020

Journal: Annals of Epidemiology and Public health

Publisher: MedDocs Publishers LLC

Online edition: http://meddocsonline.org/

Copyright: (C) Antwi GO (2020). This Article is

distributed under the terms of Creative Commons

Attribution 4.0 International License

Keywords: Electronic cigarettes; Combustible cigarette smoking; Adolescent and young adult; cancer survivors.

\section{Abstract}

Use of e-cigarettes, which contain nicotine and other harmful substances, is trending upward and posing adverse health effects for users. A review of literate demonstrated a dearth of studies regarding e-cigarettes use and its associated factors in adult Cancer Survivors, ages $\geq 18$ years, who were Adolescents or Young Adults, ages 15-39, at disease Onset (AYAO-CS). This study seeks to examine the cross-sectional relationships between e-cigarette use and combustible cigarette smoking, plus demographic characteristics, in AYAO-CS and matched controls with no history of cancer.

Using pooled data from the 2017 and 2018 Behavioral Risk Factor Surveillance System; logistics regression analysis estimated odds of e-cigarette use in a sample of 2,592 adults (1,296 AYAO-CS and 1, 296 non-cancer controls). Overall, approximately $6 \%$ reported current e-cigarette use; $6.41 \%$ and $4.38 \%$ of AYAO-CS and controls, respectively. Approximately $25 \%$ were current combustible cigarette smokers; $28.37 \%$ and $17.86 \%$ of AYAO-CS and controls, respectively. Current and former combustible cigarette smokers were more likely to be current e-cigarette users (OR=48.99, 95\% Cl: 15.53 $154.56)$ and (OR=4.41, 95\% Cl: $1.41-13.77)$ respectively.

Although AYAO-CS had greater, but non-significant, odds of current e-cigarette use (OR=1.34, 95\% Cl: 0.75-2.43) compared with non-cancer controls, findings support the need for coordinated efforts to generate greater awareness among cancer survivors of the potential adverse outcomes associated with e-cigarette use and concurrent use with combustible cigarettes, as well as counseling services regarding other proven smoking cessation strategies in lieu of e-cigarette use 
Introduction

Electronic Nicotine Delivery Systems (ENDS or e-cigarettes) entered the U.S. tobacco market in 2007 and have since seen a dramatic use prevalence increase, particularly among adolescents and young adults [1]. The e-cigarette brand JUUL, alone, recorded increased sales of approximately 600\% during 2016 2017, making it the most popular and the most commonly used e-cigarette in the U.S. [2]. The upward trend in the prevalence of e-cigarettes is not unique to the general population, as prior studies have reported similar findings in the cancer survivor subpopulation. For instance, one prior study observed a threefold increase in e-cigarette use in a sample of cancer patients enrolled in a tobacco treatment program of a comprehensive cancer center after a 12-month fellow-up period [3].

Noteworthy, significant positive associations have been reported between e-cigarette use and use of other substances, particularly combustible cigarette smoking. For example, a past study concluded that current e-cigarette use was associated with significant odds of combustible cigarette smoking among adolescents (OR=2.73, 95\% Cl: 2.00-3.73) [4]. A prior longitudinal study concluded that current e-cigarette use had an independent significant association with future cigarette smoking initiation (OR=5.48, 95\% C1:2.69-11.2) [5]. The same study found a greater than $40 \%$ combustible cigarette smoking initiation rate among ever e-cigarette users during a 16-month follow-up period [5]. Within the cancer survivor subpopulation, one study found that $38.5 \%$ of current combustible cigarette smokers reported past month e-cigarette use [3]. In a sample of cancer survivors in the U.S., a prior study observed that the odds of current cigarette smoking were significantly higher for survivors who reported ever e-cigarette use by a multiplicative factor of $11.81(95 \% \mathrm{Cl}, 5.38-25.93)$ [6]. Lastly, one study involving a large sample of 36,308 U.S. adults, recorded that daily and nondaily e-cigarette use were significantly associated with alcohol use disorder ( $\mathrm{OR}=2.5495 \% \mathrm{Cl}=1.52-4.25$ ) and (OR $=3.56$ $95 \% \mathrm{Cl}=1.24-10.26$ ) respectively, after controlling for other factors [7].

Furthermore, previous research has established that almost all e-cigarettes contain nicotine and other harmful products such as ultrafine, diacetyl $[1,8]$. Prior research further found that all e-cigarettes contain nicotine; even cartridges labeled as nicotine-free contain some amount of nicotine [8]. Over 70 eliquid brands now sell high level nicotine e-cigarette products ranging between $5 \%$ and $7 \%$, equivalent to packs of more than 40 cigarette [9]. Since evidence is mounting that ingredients in e-cigarette pose health risks to users, in general [10] it stands to reason that such use could also generate adverse health effects among those with chronic illnesses, including adults who survived cancers that were effectively treated during their adolescent and young adult years.

Although prior studies have examined e-cigarette use and its association with other substances, both in the general population and the cancer survivor subpopulation, a review of the available literature demonstrates a dearth of studies focusing on e-cigarettes use and its association with combustible cigarette smoking among adult cancer survivors, ages 18 years and older, who were first diagnosed when they were adolescents or young adults between the ages of 15 and 39 [11]. (This population is hereafter be referred to Adolescent and Young Adult Onset Cancer Survivors, using the acronym "AYAO-CS"). Therefore, the current study seeks to examine the cross-sectional relationships between combustible cigarette smoking and e-cigarette use among AYAO-CS and their age and sex matched peers with no history of cancer (Non-cancer matched controls).

\section{Methods}

\section{Data Source}

This study utilized a pooled data from the 2017 and 2018 Behavioral Risk Factor Surveillance Survey (BRFSS). BRFSS is the largest annual cross-sectional and premier national survey in the U. S. whereby a Computer-Assisted Telephone Interview (CATI) system is utilized to gather data from all the 50 states, the District of Columbia and three U.S. territories (Puerto Rico, Guam, and the U.S. Virgin Islands) [12,13]. Data collection occurs at the state levels by employing a multistage cluster sampling design based on random digit-dialing methods to collect data from noninstitutionalized U.S. civilian adults $[12,13]$.

\section{Study Sample}

The current study includes individuals with a cancer diagnosis during adolescence and young adulthood and a matched control with no history of cancer diagnosis (non-cancer controls). Participants' cancer status was determined via the survey questions: "Has a doctor, nurse or other health care professional ever told you had any other types of cancer?" (That is all other cancers except skin cancer). Those who responded "yes" were asked a follow-up question; "At what age were you told that you had cancer?" Individuals who were age $\geq 18$ years at time of survey administration and ages 15 to 39 years at the time of cancer onset were included in the current study (AYAO-CS). A one-by-one (1:1) propensity score-matched analysis, controlling for age and sex, was conducted to select the non-cancer controls. The 1:1 propensity score matching yielded matched pairs of 1,296 AYAO-CS and 1, 296 non-cancer controls.

This study was deemed exempt for review by the Institutional Review Board at Indiana University, Bloomington

\section{Measures}

Seminal to this study, prevalence of e-cigarette use and combustible cigarette smoking among both AYAO-CS and matched non-cancer controls were determined.

E-cigarette User Status was derived from two survey questions: "Have you ever used an e-cigarette or other electronic "vaping" product, even just one time, in your entire life?" and "Do you now use e-cigarettes or other electronic "vaping" products every day, some days, or not at all?" Based on responses, three categories were identified: Current-(those who use e-cigarettes currently every day or some days); former-user (those who do not use e-cigarette currently but had used it in the past); never-users (those who had never used e-cigarettes in their lifetime).

Combustible Cigarette Smoking Status was determined from two survey questions: "Have you smoked at least 100 cigarettes in your entire life?" and "Do you now smoke cigarettes every day, some days, or not at all?" Based on responses, three categories were identified: current - (those who smoke cigarettes currently every day or some days); former smokers (those who do not smoke currently but had smoked at least 100 cigarettes in their entire life); never smokers (those who had never smoked in their lifetime).

Covariates included for analysis were age, gender, race, marital status, educational level, employment status, general health 
status and past month alcohol use. All covariates were treated as categorical variables.

\section{Statistical analysis}

All analyses were weighted to account for the complex sample design. Descriptive statistics were used to summarize e-cigarette user status, smoking status, past month alcohol use and demographic characteristics by cancer status. Pearson Chisquared Test $(\chi 2)$ was conducted to determine if differences were significant. Multinomial logistic regression examined the independent associations between combustible cigarette smoking status, past month alcohol use, cancer status and e-cigarette user status adjusting for age, gender, race, marital status, educational level, employment status, and general health status. SAS version 9.4 (SAS Institute, Inc., Cary, North Carolina) was used for all statistical analyses.

\section{Results}

\section{Participants' demographics}

Table-1 provides study participant characteristics; a majority were female (73.44 \%); middle-aged (between 40- 65 years; $58.71 \%)$, Non-Hispanic Whites (80.83\%) and currently employed (52.19\%). Approximately one-fourth (25.83\%) had college degree or beyond. The total unweighted sample size for this study was 2,592(1,296 AYAO-CS and 1,296 Non-cancer controls).

The overall prevalence rates for current and former e-cigarette use were $5.89 \%$ and $19.57 \%$, respectively; while 74.54 $\%$ had never used e-cigarette in their entire life. The overall prevalence rate for current combustible cigarette smoking was $25.35 \%$; respectively, $26.10 \%$ and $48.55 \%$ were former and never-combustible cigarette smokers. Furthermore, $51.95 \%$ of the participants reported past month alcohol use.

The prevalence of current e-cigarette use and combustible cigarette smoking in AYAO-CS were $6.41 \%$ and $28.37 \%$ respectively; both were greater than for non-cancer matched controls; $4.38 \%$ and $17.86 \%$ respectively. Among AYAO-CS, $51.73 \%$ reported past month alcohol use, compared to $52.48 \%$ of noncancer matched controls.

Table 1: Participants' Demographic Characteristics, E-cigarette Use, Combustible Cigarette Smoking and Alcohol Use by Cancer Status

\begin{tabular}{|c|c|c|c|c|c|}
\hline & \multicolumn{3}{|c|}{ Overall Sample } & \multirow{2}{*}{$\begin{array}{c}\text { AYAO-CS } \\
\text { Col \% }\end{array}$} & \multirow{2}{*}{$\begin{array}{c}\text { Non-Cancer Controls } \\
\text { Col \% }\end{array}$} \\
\hline & N & Weighted & $\%$ & & \\
\hline \multicolumn{6}{|l|}{ Age } \\
\hline 18- 39 years (Young Adult) & 464 & 219518 & 28.29 & 26.26 & 33.35 \\
\hline 40-64 years (Middle-aged) & 1486 & 455534 & 58.71 & 60.68 & 53.82 \\
\hline$\geq 65$ years (Older Adult) & 642 & 100870 & 13.00 & 13.08 & 12.83 \\
\hline \multicolumn{6}{|l|}{ Gender } \\
\hline Female & 1908 & 569852 & 73.44 & 70.97 & 79.57 \\
\hline Male & 684 & 206070 & 26.56 & 29.03 & 20.43 \\
\hline \multicolumn{6}{|l|}{ Race/Ethn. } \\
\hline White & 2084 & 627170 & 80.83 & 86.61 & 66.48 \\
\hline Black & 74 & 43758 & 5.64 & 6.30 & 3.99 \\
\hline Hispanic & 363 & 72576 & 9.35 & 3.35 & 24.25 \\
\hline Other & 71 & 32418 & 4.18 & 3.74 & 5.28 \\
\hline \multicolumn{6}{|l|}{ Marital Status } \\
\hline Married & 1562 & 461905 & 59.53 & 60.43 & 57.29 \\
\hline Divorced & 534 & 126201 & 16.27 & 17.15 & 14.08 \\
\hline Single & 330 & 115094 & 14.83 & 13.15 & 19.01 \\
\hline Other & 166 & 72722 & 9.37 & 9.27 & 9.62 \\
\hline \multicolumn{6}{|l|}{ Education } \\
\hline$<$ High school & 140 & 78712 & 10.14 & 10.52 & 9.20 \\
\hline High school graduate & 696 & 218311 & 28.14 & 29.51 & 24.73 \\
\hline Some college & 768 & 278472 & 35.89 & 34.87 & 38.43 \\
\hline College or more & 988 & 200427 & 25.83 & 25.20 & 27.65 \\
\hline \multicolumn{6}{|l|}{ Employment Status } \\
\hline Employed & 1379 & 422567 & 54.46 & 52.19 & 60.10 \\
\hline Unemployed & 121 & 52930 & 6.82 & 7.49 & 5.15 \\
\hline
\end{tabular}


MedDocs Publishers

\begin{tabular}{|c|c|c|c|c|c|}
\hline Homemaker & 146 & 56177 & 7.24 & 7.18 & 7.38 \\
\hline Other & 946 & 244249 & 31.48 & 33.13 & 27.37 \\
\hline \multicolumn{6}{|c|}{ General Health Status } \\
\hline Excellent & 339 & 81523 & 10.51 & 7.54 & 17.88 \\
\hline Fair & 441 & 130224 & 16.78 & 17.47 & 15.08 \\
\hline Poor & 213 & 86380 & 11.13 & 14.36 & 3.12 \\
\hline \multicolumn{6}{|c|}{ E-cigarette User Status } \\
\hline Current-User & 101 & 45664 & 5.89 & 6.49 & 4.38 \\
\hline Never-Never & 2068 & 578416 & 74.54 & 72.99 & 78.42 \\
\hline \multicolumn{6}{|c|}{ combustible cigarette smoking Status } \\
\hline Current-Smoker & 531 & 196702 & 25.35 & 28.37 & 17.86 \\
\hline Former-Smoker & 747 & 202512 & 26.1 & 26.72 & 24.55 \\
\hline Never-Smoker & 1314 & 376708 & 48.55 & 44.91 & 57.6 \\
\hline \multicolumn{6}{|c|}{ Past Month Alcohol Use } \\
\hline No & 1225 & 372863 & 48.05 & $48.27 \%$ & $47.52 \%$ \\
\hline Yes & 1367 & 403059 & 51.95 & $51.73 \%$ & $52.48 \%$ \\
\hline
\end{tabular}

Table 2 presents the crude associations between e-cigarette use and combustible cigarette smoking as well as all other independent variables. Among current e-cigarette users, $76.43 \%$ reported current cigarette smoking compared with $11.90 \%$ of never e-cigarette users who reported current combustible cigarette smoking. Among current e-cigarette users, 78.63\% were AYAO-CS and $21.37 \%$ were non-cancer matched controls.

Table 2: Unadjusted Associations between E-cigarette Use, Demographic Characteristics, Combustible Cigarette Smoking and Alcohol Use

\begin{tabular}{|c|c|c|c|c|}
\hline & Current e-cig user & Former e-cig user & Never e-cig user & P-value \\
\hline Age & weighted \% & weighted \% & weighted \% & \\
\hline Young Adult & 45.84 & 37.59 & 24.47 & \\
\hline Middle-aged & 49.43 & 57.64 & 59.72 & $<.0001$ \\
\hline Older Adult & 4.73 & 4.77 & 15.81 & \\
\hline \multicolumn{5}{|l|}{ Gender } \\
\hline Female & 82.87 & 74.5 & 72.42 & 0.328 \\
\hline Male & 17.13 & 25.5 & 27.58 & \\
\hline \multicolumn{5}{|l|}{ Race/Ethn. } \\
\hline White & 80.50 & 79.84 & 81.12 & \\
\hline Black & 1.42 & 3.84 & 6.45 & \\
\hline Hispanic & 11.39 & 12.49 & 8.37 & 0.386 \\
\hline Other & 6.69 & 3.84 & 4.07 & \\
\hline \multicolumn{5}{|l|}{ Marital Status } \\
\hline Married & 48.55 & 38.85 & 65.83 & \\
\hline Divorced & 10.52 & 29.21 & 13.32 & $<.0001$ \\
\hline Single & 19.18 & 20.44 & 13.02 & \\
\hline Other & 21.75 & 11.50 & 7.84 & \\
\hline
\end{tabular}


MedDocs Publishers

\begin{tabular}{|c|c|c|c|c|}
\hline$<$ High school & 9.60 & 13.18 & 9.39 & \\
\hline High school graduate & 41.34 & 34.08 & 25.53 & $<.0001$ \\
\hline Some college & 41.72 & 40.01 & 34.35 & \\
\hline College or more & 7.34 & 12.73 & 30.73 & \\
\hline \multicolumn{5}{|l|}{ Employment Status } \\
\hline Employed & 48.07 & 44.21 & 57.66 & \\
\hline Unemployed & 13.11 & 12.84 & 4.74 & 0.002 \\
\hline Homemaker & 11.22 & 5.69 & 7.33 & \\
\hline Other & 27.59 & 37.26 & 30.27 & \\
\hline \multicolumn{5}{|l|}{ General Health Status } \\
\hline Excellent & 7.82 & 5.62 & 12.00 & \\
\hline Good & 55.34 & 50.35 & 65.02 & $<.0001$ \\
\hline Fair & 27.07 & 24.05 & 14.06 & \\
\hline Poor & 9.76 & 19.97 & 8.92 & \\
\hline \multicolumn{5}{|l|}{ Cancer Status } \\
\hline AYAO-CS & 78.63 & 74.77 & 69.8 & 0.126 \\
\hline Non-Cancer Controls & 21.37 & 25.23 & 30.20 & \\
\hline \multicolumn{5}{|c|}{ Combustible Cigarette Smoking Status } \\
\hline Current-Smoker & 76.43 & 61.23 & 11.90 & \\
\hline Former-Smoker & 14.53 & 21.89 & 28.12 & $<.0001$ \\
\hline Never-Smoker & 9.04 & 16.88 & 59.98 & \\
\hline \multicolumn{5}{|c|}{ Past Month Alcohol Use } \\
\hline No & 43.74 & 53.4 & 46.99 & 0.326 \\
\hline Yes & 56.26 & 46.60 & 53.01 & \\
\hline
\end{tabular}

The multivariable logistics regression model predicting e-cigarette user status is presented in Table 3. The "never-users" group served as the reference category for e-cigarette use.

Table 3: Adjusted Association between E-cigarette Use and Combustible Cigarette Smoking

\begin{tabular}{|c|c|c|c|c|}
\hline & \multicolumn{2}{|c|}{ Current E-cigarette User } & \multicolumn{2}{|c|}{ Former E-cigarette User } \\
\hline & OR & $95 \% \mathrm{Cl}$ & OR & $95 \% \mathrm{Cl}$ \\
\hline \multicolumn{5}{|c|}{ Combustible Cigarette Smoking } \\
\hline Never-Smoker & 1 & - & 1 & \\
\hline Current-Smoker & 48.99 & $15.53-154.56$ & 15.97 & $9.38-27.19$ \\
\hline Former-Smoker & 4.41 & $1.41-13.77$ & 3.07 & $1.76-5.37$ \\
\hline \multicolumn{5}{|l|}{ Cancer Status } \\
\hline Control & 1 & - & 1 & \\
\hline AYAO-CS & 1.34 & $0.74-2.43$ & 0.97 & $0.64-1.49$ \\
\hline \multicolumn{5}{|l|}{ Age } \\
\hline Older & 1 & - & 1 & - \\
\hline Middle-aged & 2.70 & $1.09-6.66$ & 4.06 & $2.33-7.10$ \\
\hline Young Adults & 5.26 & $2.02-15.80$ & 7.94 & $3.51-17.98$ \\
\hline
\end{tabular}


MedDocs Publishers

\begin{tabular}{|c|c|c|c|c|}
\hline Female & 1 & - & 1 & - \\
\hline Male & 0.77 & $0.37-1.59$ & 1.11 & $0.68-1.83$ \\
\hline \multicolumn{5}{|l|}{ Race/Ethnicity } \\
\hline White & 1 & - & 1 & - \\
\hline Black & 0.24 & $0.02-2.62$ & 0.48 & $0.12-1.97$ \\
\hline Hispanic & 0.95 & $0.29-3.11$ & 1.07 & $0.57-2.01$ \\
\hline Other & 1.71 & $0.55-5.30$ & 1.07 & $0.28-4.19$ \\
\hline \multicolumn{5}{|l|}{ Marital Status } \\
\hline Married & 1 & - & 1 & - \\
\hline Divorced & 0.81 & $0.33-1.96$ & 2.57 & $1.49-4.45$ \\
\hline Single & 1.71 & $0.67-4.40$ & 2.08 & $0.99-4.34$ \\
\hline Other & 2.37 & $0.88-6.39$ & 1.35 & $0.67-2.75$ \\
\hline \multicolumn{5}{|l|}{ Educational Level } \\
\hline$<$ High school & 1 & - & 1 & - \\
\hline High school graduate & 2.42 & $0.74-7.95$ & 1.57 & $0.72-3.43$ \\
\hline Some college & 3.43 & $1.07-11.04$ & 2.34 & $1.10-5.00$ \\
\hline College or more & 1.11 & $0.27-4.41$ & 1.4 & $0.62-3.16$ \\
\hline \multicolumn{5}{|l|}{ Employment Status } \\
\hline Employed & 1 & - & 1 & - \\
\hline Unemployed & 1.26 & $0.44-3.60$ & 1.27 & $0.55-2.97$ \\
\hline Homemaker & 2.39 & $0.71-8.07$ & 1.24 & $0.58-2.66$ \\
\hline Other & 1.17 & $0.52-2.64$ & 1.34 & $0.73-2.47$ \\
\hline \multicolumn{5}{|l|}{ General Health Status } \\
\hline Excellent & 1 & - & 1 & - \\
\hline Good & 0.73 & $0.21-2.62$ & 1.14 & $0.58-2.25$ \\
\hline Fair & 0.99 & $0.24-4.02$ & 1.57 & $0.69-3.54$ \\
\hline Poor & 0.39 & $0.07-2.20$ & 1.32 & $0.47-3.74$ \\
\hline \multicolumn{5}{|c|}{ Past Month Alcohol Use } \\
\hline No & 1 & - & 1 & - \\
\hline Yes & 2.02 & $1.02-4.08$ & 1.15 & $0.74-1.81$ \\
\hline
\end{tabular}

\section{Current E-Cigarette user vs never E-Cigarette user}

Participants who reported current and former combustible cigarette smoking had significantly greater odds of being current e-cigarette users, compared with never-combustible cigarette smokers (OR=48.99, 95\% $\mathrm{Cl}: 15.53-154.56)$ and $(\mathrm{OR}=4.41$, 95\% Cl: 1.41-13.77) respectively. Although, AYAO-CS have greater odds of being current e-cigarette users compared with non-cancer controls, the difference was not statistically significant. Participants who reported past month alcohol use had significantly greater odds of being current e-cigarette users than those who had not used alcohol in the past month $(\mathrm{OR}=2.02$, 95\% Cl: 1.02-4.08). Moreover, young adults (OR=5.62, 95\% Cl: 2.02-15.80); middle-aged adults (OR=2.70, 95\% Cl: $1.09-15.80)$ and participants with some college education (OR=3.43, $95 \% \mathrm{Cl}$ : 1.07-11.04) were more likely to be current e-cigarette users.

\section{Former E-Cigarette use vs never E-Cigarette use}

After controlling for all other variables, the odds of being a former e-cigarette user were significantly greater for current combustible cigarette smokers (OR=15.97, 95\% Cl: 9.38-27.19) and former e-cigarette users (OR=3.07, 95\% Cl: 1.76-5.37) than never combustible cigarette smokers. Likewise, young adults ( $\mathrm{OR}=7.94,95 \% \mathrm{Cl}$ : 3.51-17.98); middle-aged adults (OR=4.06, 95\% Cl: 2.33-7.10); divorced participants (OR=2.57, 95\% Cl: $1.49-4.45)$ and those with some college education (OR=2.34, 95\% Cl: 1.10-5.00), were significantly more likely to be former e-cigarette users. 


\section{Discussion}

The primary objective of this study was to examine the association between e-cigarette use and combustible-cigarette smoking among AYAO-CS and matched controls with no history of cancer. To the best of the authors' knowledge, this is the first study that has examined these associations among AYAO-CS in comparison to never-cancer controls using national representative data. Study findings demonstrate that, in both AYAO-CS and matched control groups, current and former cigarette smokers were significantly more likely to use e-cigarette. For instance, the crude analysis, found that nearly eight of every ten current e-cigarette users reported currently smoking combustible cigarettes. The strong and positive association between e-cigarette use and combustible cigarette was consistent with findings from prior studies both in the general population $[4,5,14]$ and among the cancer survivor subpopulation $[3,15,16]$. For instance, a study reported that among cancer survivors who were current cigarette smokers, $34.3 \%$ reported ever e-cigarette use [15]. In the same study, current cigarette smoking independently had significant associations with higher odds of e-cigarette use $(\mathrm{OR}=8.46,95 \% \mathrm{Cl}=3.69,19.35)$ [15]. In a sample of 2, 448 U.S. adults who reported current e-cigarette users, Patel et al. [14] estimated that $92.6 \%$ were also current cigarette smokers. Additionally, recent longitudinal studies have determined significant cause and effect relationships between e-cigarette use and combustible cigarette smoking both in the general population and among the cancer survivor subpopulation [3,17]; a prospective study involving cancer patients determined that, relative to non- e-cigarette users, the odds of initiating combustible cigarette smoking were significantly greater for e-cigarette users (OR=5.23, 95\% Cl: 2.91-9.41) [3].

The high rate of concurrent combustible cigarette and e-cigarette use among participants in this study is very concerning since these behaviors could potentially result in adverse health outcomes. The American Association for Cancer Research has argued that concurrent use of e-cigarettes and combustible cigarettes poses serious threats to health, [18] including cancer recurrence or developing additional primary cancers of the esophagus, larynx, kidney, liver, or stomach, etc. [19]. Moreover, the effects could be far more detrimental for cancer survivors who engage in such behavior. Although evidence regarding the long-term effects of e-cigarette is scanty, limited available epidemiological data suggest that e-cigarette use also can potentially result in cardiovascular issues associated with nicotine addiction $[1,20,21]$. For example, a prior study using national representative data, determined that daily use of e-cigarettes was positively correlated with higher odds of developing myocardial infarction among U.S. adults $(\mathrm{OR}=1.79,95 \% \mathrm{Cl}=1.20$, 2.66, $p=0.004$ ) [22]. Furthermore, sufficient data have linked acute administration of nicotine to a number of adverse outcomes including increases in heart rate and blood pressure as well as respiratory and gastrointestinal disorders [1,23].

Some of the most common cancers in adolescents and young adults, including breast cancer, cervical and thyroid, [24] are relatively more prevalent in women; this circumstance could possibly explain the disproportionate representation of women (73.44\%) in this study. Regarding demographic characteristics consistent with previous research, young adults were relatively more likely to report current e-cigarettes use [6]. Additionally, high rate of current e-cigarette use were observed among participants with some college education and divorced participants.
Thus, intervention design programs need to prioritize individuals with such characteristics.

Available studies identified easy access, low cost of e-cigarette, low perceived harm, less stigmatization, less toxicity than combustible cigarettes, social attractiveness, flavoring and much higher nicotine content in newer e-cigarettes as key contributing factors to the increased trend in e-cigarette use $[16,25,27]$. Particularly, a recent study that examined cancer patients' beliefs about e-cigarette use recorded that patients use e-cigarette for different reasons including; managing cancer-related stress, less likelihood of interfering with cancer treatment and use as a combustible cigarette smoking cessation aid [16]. Consistent with the last point, studies in the general population have concluded that using e-cigarette for quitting combustible cigarette smoking partly accounts for the high rate of the concurrent use of both substances $[28,29]$. Nevertheless, a study that examined the influence of e-cigarette use on quitting combustible cigarette smoking concluded that e-cigarettes only had moderately positive effects on helping smokers quit, with no statistical evidence of superiority over other cessation strategies such as nicotine replacement therapy [30].

Another interesting and quite troubling finding from the current study is the higher prevalence of both current e-cigarettes use and combustible cigarette smoking among AYAO-CS compared with the never-cancer control group. This finding highlights the urgent need for sustained availability and accessibility of tobacco cessation programs for AYAO-CS. Currently, few if any, studies have been conducted to determine the short and long-term effects of e-cigarette use among cancer patients and/or survivors. This lack of research could possibly contribute to the high prevalence of e-cigarette use in this unique at-risk population.

\section{Implications}

This study is the first to examine the association between combustible cigarette smoking and e-cigarette use among AYAO-CS with matched controls, using a national representative data. The findings can contribute substantially to the limited literature on e-cigarette use among cancer survivors and serve as justification for implementation of effective behavioral, environmental and policy interventions aimed at reducing e-cigarette use, combustible cigarette smoking and/or concurrent use of both products. First, the strong significant positive association between use of the two products and the previously identified potential detrimental health effects associated with concurrent use of e-cigarettes and combustible cigarettes justify the need for a more comprehensive approach to prevention of substitute use and/or dual use of both, particularly among AYAO-CS. Specific strategies such as mass media campaigns, high taxation of e-cigarette products and raising the legal purchase and use age as well as creating more awareness about the potential harmful effects of e-cigarette use, can help protect the health of AYAOCS who are already at greater risk for other negative health conditions including cardiovascular disease, obesity, poor physical and mental health [31].

The higher rate of e-cigarette use among AYAO-CS in the current study and the low likely perception of harm associated with e-cigarette use suggest the need for creating awareness about the potential detrimental effects of e-cigarette use alone as well as concurrently with combustible cigarettes, particularly among AYAO-CS. 
Moreover, long-term counselling services are needed for cancer survivors who are combustible cigarette smokers to educate them regarding effective and proven smoking cessation strategies besides e-cigarettes, as this may help prevent the concurrent use of both products. Specific strategies such as nicotine replacement therapy, motivational interviewing, physician-based counseling and peer-based counselling [1,32,33] have proven effective with both the general population and the cancer survivor subpopulation. Because higher rates of e-cigarettes use and concurrent use of both products were recorded among AYAO-CS less than 65 years of age; thus, intervention designers should prioritize young and middle-aged adults.

Additional new approaches that apply health behavior theories such as Protection Motivation Theory (PMT) should also be considered. PMT has been shown to significantly influence substance use behaviors in the general population [34] [35]; for example, one study reported that adolescents' smoking behaviors were significantly associated with both threat and coping appraisals, after adjusting for other covariates [34]. A second study found that, relative to adolescents with higher perceived smoking-related health risks, those with lower perceived risks had 3.64 times greater the odds of initiating cigarette smoking [36]. Therefore, future studies can incorporate health behavior theories to examine whether AYAO-CS' threat and coping appraisal skills moderate the associations between combustible cigarette smoking and e-cigarette use. Lastly, more longitudinal data are needed to examine both the short and long-term effects of e-cigarette use on cancer survivorship and overall quality of health.

\section{Limitations}

A key limitation of the current study is the cross-sectional nature of the data which limits the authors' ability to draw causal conclusions about the association between combustible cigarette smoking and e-cigarette use. Secondly, data on combustible cigarette smoking and e-cigarette use were gathered through self-reporting and, thus, could potentially suffer from social desirability bias. Nevertheless, a prior study reported that questions relating to self-reported cigarette smoking behaviors demonstrated high agreement (Kappa $=0.81$ 95\% Cl 0.72-0.89), respectively [37]. Additionally, previous research justifies the assumption that alcohol and tobacco use behavior self-reported by the participants were reliable and reflected their true behavior [38]. Another limitation, not unique to the current study, is the under representation of minority race/ethnicity and individuals with lower socioeconomic status in national surveys [39] [40]. Lastly, though the sample is a true representative of the AYAO-CS population in the United States, the generalizability of the findings could vary by state due to differences in state-level response rate $[12,13]$, plus differences in tobacco control policies and programs including taxation rates.

\section{Conclusion}

Among AYAO-CS and matched controls, current and former combustible cigarette smoking was associated with higher odds of e-cigarette use, after adjusting for other variables. Young and middle-aged adults in both groups were more likely to engage in concurrent use of both products, highlighting the need for intervention designers to prioritize these sub-groups. Finally, study findings suggest the need for coordinated efforts to generate greater awareness of the potential adverse outcomes associated with e-cigarette use and concurrent use of both products as well as counseling services for combustible cigarette smokers with history of cancer diagnosis on proven effective smoking cessation strategies.

\section{References}

1. US Department of Health and Human Services. E-Cigarette Use Among Youthand Young Adults: A Report of the Surgeon General. Atlanta, GA: US Department of Health and Human Services, Centers for Disease Control and Prevention, National Center for Chronic Disease Prevention and Health Promotion, Office on Smoking and Health. 2016.

2. King BA, Gammon DG, Marynak KL, Rogers T. Electronic Cigarette Sales in the United States. 2013-2017. Jama. 2018; 320: 1379.

3. Borderud SP, Li Y, Burkhalter JE, Sheffer CE, Ostroff JS. Electronic cigarette use among patients with cancer: Characteristics of electronic cigarette users and their smoking cessation outcomes. Cancer. 2014; 120: 3527-3535.

4. Leventhal AM, Strong DR, Kirkpatrick MG, et al. Association of Electronic Cigarette Use With Initiation of Combustible Tobacco Product Smoking in Early Adolescence. Jama. 2015; 314: 700.

5. Barrington-Trimis JL, Urman R, Berhane K, et al. E-Cigarettes and Future Cigarette Use. American Academy of Pediatrics. June 2016.

6. Antwi GO, Lohrmann DK, Jayawardene W, Chow A, Obeng CS. et al. Associations between e-cigarette and combustible cigarette use among U.S. cancer survivors: implications for research and practice. Journal of Cancer Survivorship. 2019; 13: 316-325.

7. Roberts W, Moore KE, Peltier MR, et al. Electronic Cigarette Use and Risk of Harmful Alcohol Consumption in the U.S. Population. Alcoholism: Clinical and Experimental Research. 2018; 42: 2385-2393.

8. Clearing the smoke. Nature Neuroscience. 2014; 17: 1013-1013.

9. Jackler RK, Ramamurthi D. Nicotine arms race: JUUL and the high-nicotine product market. Tobacco Control. 2019.

10. Outbreak of Lung Injury Associated with E-Cigarette Use, or Vaping. Centers for Disease Control and Prevention. 2019.

11. Adolescent and Young Adult Oncology Progress Review Group. Closing the Gap: Research and Care Imperatives for Adolescents and Young Adults with Cancer. NIH Pub. No. 06-6067. Bethesda, MD: National Cancer Institute, National Institutes of Health, and the LIVESTRONG Young Adult Alliance; 2006.

12. Centers for Disease Control and Prevention. Behavioral Risk Factor Surveillance System OVERVIEW: BRFSS 2017. July 2018.

13. Centers for Disease Control and Prevention. Behavioral Risk Factor Surveillance System OVERVIEW: BRFSS 2018. July 2019.

14. Patel D, Davis KC, Cox S, et al. Reasons for current E -cigarette use among U.S. adults. Preventive Medicine. 2016; 93: 14-20. 
15. Salloum RG, Getz KR, Tan AS, et al. Use of Electronic Cigarettes among Cancer Survivors in the U.S. American Journal of Preventive Medicine. 2016; 51: 762-766.

16. Correa JB, Brandon KO, Meltzer LR, et al. Electronic cigarette use among patients with cancer: Reasons for use, beliefs, and patient-provider communication. Psycho-Oncology. 2018; 27: 1757-1764.

17. East K, Hitchman SC, Bakolis I, et al. The Association Between Smoking and Electronic Cigarette Use in a Cohort of Young People. Journal of Adolescent Health. 2018; 62(5): 539-547.

18. Toll BA, Brandon TH, Gritz ER, Warren GW, Herbst RS. Assessing Tobacco Use by Cancer Patients and Facilitating Cessation: An American Association for Cancer Research Policy Statement. Clinical Cancer Research. 2013; 19: 1941-1948.

19. U.S. Department of Health and Human Services. Let's Make the Next Generation Tobacco-Free: Your Guide to the 50th Anniversary Surgeon General's Report on Smoking and Health. Atlanta: U.S. Department of Health and Human Services, Centers for Disease Control and Prevention, National Center for Chronic Disease Prevention and Health Promotion, Office on Smoking and Health. 2014.

20. Benowitz NL, Burbank AD. Cardiovascular toxicity of nicotine: Implications for electronic cigarette use. Trends in Cardiovascular Medicine. 2016; 26:515-523.

21. Bhatnagar A. Cardiovascular Perspective of the Promises and Perils of E-Cigarettes. Circulation Research. 2016; 118: $1872-1875$.

22. Alzahrani T, Pena I, Temesgen N, Glantz SA. Association Between Electronic Cigarette Use and Myocardial Infarction. American Journal of Preventive Medicine. 2018; 55: 455-461.

23. Chaturvedi P, Mishra A, Datta S, Sinukumar S, Joshi P, Garg A. Harmful effects of nicotine. Indian Journal of Medical and Paediatric Oncology. 2015; 36: 24.

24. Adolescents and Young Adults (AYAs) with Cancer. National Cancer Institute. 2019.

25. Saddleson M, Kozlowski L, Giovino G, et al. Enjoyment and other reasons for electronic cigarette use: Results from college students in New York. Addictive Behaviors. 2016; 54: 33-39.

26. Simonavicius E, Mcneill A, Arnott D, Brose LS. What factors are associated with current smokers using or stopping ecigarette use? Drug and Alcohol Dependence. 2017; 173: 139-143.

27. Villanti AC, Johnson AL, Ambrose BK, et al. Flavored Tobacco Product Use in Youth and Adults: Findings From the First Wave of the PATH Study (2013-2014). American Journal of Preventive Medicine. 2017; 53: 139-151.

28. Xu Y, Guo Y, Liu K, Liu Z, Wang X. E-Cigarette Awareness, Use, and Harm Perception among Adults: A Meta-Analysis of Observational Studies. Plos One. 2016; 11.
29. Pasquereau A, Guignard R, Andler R, Nguyen-Thanh V. Electronic cigarettes, quit attempts and smoking cessation: A 6-month follow-up. Addiction. 2017; 112: 16201628.

30. Bullen C, Howe C, Laugesen M. Electronic Cigarettes for Smoking Cessation: A Randomised Controlled Trial. Journal of Vascular Surgery. 2014; 59: 872.

31. Tai E, Buchanan N, Townsend J, Fairley T, Moore A, Richardson LC. Health status of adolescent and young adult cancer survivors. Cancer. 2012; 118: 4884-4891.

32. Emmons KM, Puleo E, Park E, et al. Peer-Delivered Smoking Counseling for Childhood Cancer Survivors Increases Rate of Cessation: The Partnership for Health Study. Journal of Clinical Oncology. 2005; 23: 6516-6523.

33. Heckman CJ, Egleston BL, Hofmann MT. Efficacy of motivational interviewing for smoking cessation: a systematic review and meta-analysis. Tobacco Control. 2010; 19: 410-416.

34. Xu Y, Chen X. Protection motivation theory and cigarette smoking among vocational high school students in China: a cusp catastrophe modeling analysis.Global Health Research and Policy.2016; 1: 3.

35. Yan Y, Jacques-Tiura AJ, Chen X, et al. Application of the Protection Motivation Theory in Predicting Cigarette Smoking Among Adolescents in China. Addictive behaviors. 2014; 39: 181-188.

36. Song AV, Morrell HER, Cornell JL, et al. Perceptions of Smoking-Related Risks and Benefits as Predictors of Adolescent Smoking Initiation. American Journal of Public Health. 2009; 99: 487-492.

37. Grande ED, Fullerton S, Taylor AW. Reliability of self-reported health risk factors and chronic conditions questions collected using the telephone in South Australia, Australia. BMC Medical Research Methodology. 2012; 12.

38. Lintonen T. The Reliability Of Self-Reported Drinking In Adolescence. Alcohol and Alcoholism. 2004; 39: 362-368.

39. Voigt LF. Characteristics of Telephone Survey Respondents According to Willingness to Participate. American Journal of Epidemiology. 2003; 157: 66-73.

40. Link MW, Mokdad AH, Stackhouse HF, et al. Race, ethnicity, and linguistic isolation as determinants of participation in public health surveillance surveys. Prev Chronic Dis 2006; 3: A09 